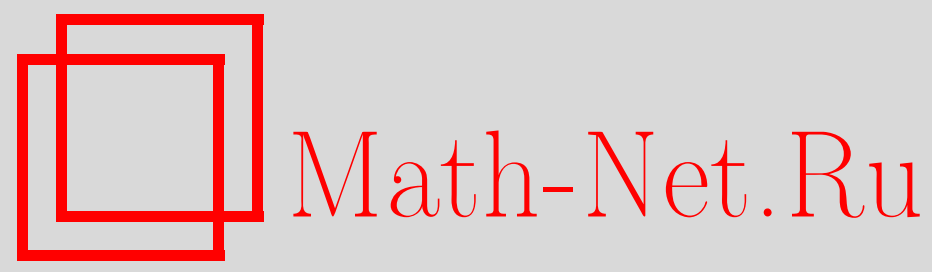

Г. И. Ибрагимов, О задачах линейной дискретной игры преследования, Матем. заметки, 2005, том 77, выпуск 5, 707718

DOI: https://doi.org/10.4213/mzm2526

Использование Общероссийского математического портала Math-Net.Ru подразумевает, что вы прочитали и согласны с пользовательским соглашением http://www. mathnet.ru/rus/agreement

Параметры загрузки:

IP: 18.207 .199 .55

26 апреля 2023 г., 14:26:25

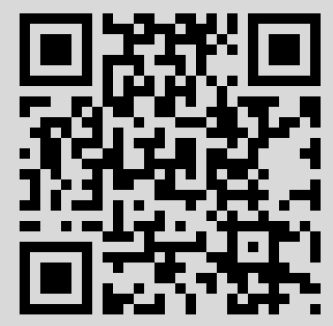




\section{О ЗАДАЧАХ ЛИНЕЙНОЙ ДИСКРЕТНОЙ ИГРЫ ПРЕСЛЕДОВАНИЯ}

\section{Г.И. Ибрагимов}

В данной работе рассматриваются две задачи линейной дискретной игры преследования. В каждой из них члены последовательности, определяющей управление преследующего, ограничены некоторым заданным положительным числом. В первой задаче члены последовательности, определяющей управление убегающего, ограничены некоторым положительным числом, а во второй задаче - сумма $p$-х степеней членов этой последовательности ограничена некоторым заданным числом. Для каждой задачи получено необходимое и достаточное условие для возможности завершения преследования из всех точек пространства.

Библиографоия: 14 названий.

1. Постановка задачи. Изучению дифференциальных и дискретных игр преследования посвящено много работ (например, [1]-[12]). Ранее [8], [9] были получены достаточные условия для завершения игры с интегральньми ограничениями и для ее дискретного аналога.

В данной работе рассматриваются две задачи линейной дискретной игры преследования. В каждой из них на управление преследующего наложено геометрическое ограничение, а на управление убегающего в первой задаче - геометрическое ограничение, и во второй задаче - суммарное ограничение.

Для каждой задачи получено необходимое и достаточное условие для возможности завершения преследования из всех точек пространства.

Работа продолжает исследования [6], [8], [9] и [11].

Пусть дискретная система описьвается рекуррентными уравнениями

$$
z(k+1)=C z(k)-u(k)+v(k)
$$

где $z, u, v \in \mathbb{R}^{n}, n \geqslant 1, k$ - номер шага, $k=1,2, \ldots, C$ - постоянная квадратная матрица порядка $n, u$ - управляющий параметр преследования; $v$ - управляющий параметр убегания. Параметр $u$ выбирается в виде последовательности

$$
u=u(\cdot)=(u(1), u(2), \ldots, u(k), \ldots), \quad|u(k)| \leqslant \rho,
$$

где $\rho$ - заданное положительное число. Параметр $v$ выбирается в виде последовательности

$$
v=v(\cdot)=(v(1), v(2), \ldots, v(k), \ldots)
$$


При этом рассматриваются два вида ограничений:

1) геометрическое ограничение

$$
|v(k)| \leqslant \sigma, \quad k=1,2, \ldots
$$

2) суммарное ограничение

$$
\|v(\cdot)\|=\left(\sum_{k=1}^{\infty}|v(k)|^{p}\right)^{1 / p} \leqslant \sigma
$$

где $p, \sigma$ - заданныеположительные числа. Таким образом, в этом случаепараметр $v$ выбирается в виде последовательности $v=v(\cdot)=(v(1), v(2), \ldots, v(k), \ldots)$ из замкнутого шара $S(\sigma)$ радиуса $\sigma$ с центром в начале координат пространства $l_{p}$. В ограничении $(2)$ предполагается, что число $\sigma$ удовлетворяет неравенству $\sigma<\rho$, а в ограничении (3) выполнение этого неравенства не требуется.

Игра (1), (2) (соответственно (1), (3)) считается завершенной, если $z(r)=0$ для некоторого значения $r$ номера $k$.

ОПРЕДЕЛЕНИЕ 1. Будем говорить, что в игре (1), (2) (соответственно (1), (3)) из начального положения $z_{0}$ возможно завершение преследования за $N\left(z_{0}\right)$ шагов, если по любой последовательности $v(\cdot)$, удовлетворяющей ограничению (2) (соответственно (3)), можно построить такую последовательность $u(\cdot)=(u(1), u(2), \ldots, u(k), \ldots)$, $|u(k)| \leqslant \rho$, что решение $z=z(\cdot)=\{z(1), z(2), \ldots, z(k), \ldots\}$ уравнения

$$
z(k+1)=C z(k)-u(k)+v(k), \quad z(1)=z_{0},
$$

удовлетворяет условию $z(\bar{k})=0$, где $1 \leqslant \bar{k} \leqslant N\left(z_{0}\right)$. При этом для нахождения значения $u(k)$ параметра $u$ на $k$-м шаге, $k \geqslant 1$, разрешается использовать $z(1), z(2), \ldots, z(k)$, $v(k)$.

2. Решение задачи 1. В этом разделе дается решение задачи 1 . Имеет место следующая теорема.

ТЕОрема 1. Если все собственные числа матрицы $С$ по модулю не превосходят единиць, то в игре (1), (2) из любого начального положения $z_{0}$ возможно завершение преследования за некоторое конечное число шагов $N\left(z_{0}\right)$.

Рассмотрим управляемый дискретный процесс, описьваемьй уравнением

$$
z(k+1)=C z(k)-\bar{w}(k), \quad z(1)=z_{0},
$$

где $z \in \mathbb{R}^{n}, k=1,2, \ldots$, управляющий параметр $\bar{w}$ выбирается в виде последовательности $\bar{w}=\bar{w}(\cdot)=(\bar{w}(1), \bar{w}(2), \ldots, \bar{w}(k), \ldots),|\bar{w}(k)| \leqslant \rho-\sigma$.

ОПРЕДЕЛЕНИЕ 2. Будем говорить, что в системе (4) из начального положения $z_{0}$ возможен перевод фазовой точки z в начало координат за $N\left(z_{0}\right)$ шагов, если можно построить такую последовательность $\bar{w}(\cdot),|\bar{w}(k)| \leqslant \rho-\sigma$, что решение $z=z(\cdot)$ уравнения (4) с начальным условием $z(1)=z_{0}$ удовлетворяет условию $z(\bar{k})=0$, где $1 \leqslant \bar{k} \leqslant N\left(z_{0}\right)$. 
Лемма 1. Если все собственные числа матричы $C$ по модулю не превосходят единицы, то в системе (4) из любого начального положения $z_{0}$ возможен перевод фазовой точки z в начало координат за некоторое число шагов $N\left(z_{0}\right)$.

ДоКАЗАТЕЛЬСТво. С помощью неособенного действительного преобразования $z=S x$ систему (4) можно привести к следующему виду:

$$
x(k+1)=J x(k)-w(k), \quad w(k)=S^{-1} \bar{w}(k), \quad x(1)=x_{0}=S^{-1} z_{0},
$$

где $J=\operatorname{diag}\left\{J_{0}, J_{1}, \ldots, J_{l_{1}}, B_{0}, B_{1}, \ldots, B_{l_{2}}\right\}$ (см. приложение). Предполагается, что $|w(k)| \leqslant \bar{\rho}$, где $\bar{\rho}$ - некоторое положительное число, которое выбирается ниже. Система (5) естественньм образом распадается на следующие системы:

$$
\begin{aligned}
& x_{1}^{i}(k+1)=J_{i} x_{1}^{i}(k)-w_{1}^{i}(k), \quad i=0,1, \ldots, l_{1}, \\
& x_{2}^{j}(k+1)=B_{j} x_{2}^{j}(k)-w_{2}^{j}(k), \quad j=0,1, \ldots, l_{2},
\end{aligned}
$$

где $x_{1}^{i}$ и $w_{1}^{i}$ (соответственно $x_{2}^{j}$ и $\left.w_{2}^{j}\right)$ - векторы, составленные из координат векторов $x$ и $w$, соответствуюших клетке Жордана $J_{i}$ (соответственно $B_{j}$ ).

Покажем возможность перевода фазовой точки $x_{1}^{i}$ в начало координат.

1. Система, соответствуюшая клетке $J_{0}$. Система уравнений

$$
x_{1}^{0}(k+1)=J_{0} x_{1}^{0}(k)-w_{1}^{0}(k)
$$

распадается на скалярные уравнения вида

$$
y(k+1)=\lambda y(k)-w(k) .
$$

Отсюда имеем

$$
y(k+1)=\lambda^{k} y(1)-\left(\lambda^{k-1} w(1)+\cdots+\lambda w(k-1)+w(k)\right) .
$$

А. Случай, когда $|\lambda|<1$. Тогда для некоторого номера $k^{0}$ имеем $\left|\lambda^{k^{0}} y(1)\right| \leqslant \bar{\rho}$. Полагаем

$$
w(k)= \begin{cases}\lambda^{k^{0}} y(1), & k=k^{0}, \\ 0, & k \neq k^{0} .\end{cases}
$$

Тогда из (9) вытекает

$$
y\left(k^{0}+1\right)=\lambda^{k^{0}} y(1)-w\left(k^{0}\right)=0
$$

и, следовательно, $y(k)=0, k \geqslant k^{0}+1$.

Б. Случай, когда $\lambda=1$. Тогда согласно (9) имеем

$$
y(k+1)=y(1)-(w(1)+\cdots+w(k-1)+w(k)) .
$$


Номер $k^{0}$ выберем так, чтобы $|y(1)| \leqslant k^{0} \bar{\rho}$. Полагаем

$$
w(k)= \begin{cases}y(1) / k^{0}, & k=1,2, \ldots, k^{0} \\ 0, & k \geqslant k^{0}+1 .\end{cases}
$$

Тогда согласно (10) имеем

$$
y\left(k^{0}+1\right)=y(1)-\frac{y(1)}{k^{0}} k^{0}=0 .
$$

В. Случай, когда $\lambda=-1$. Тогда согласно (9) имеем

$$
y(k+1)=(-1)^{k} y(1)-\left((-1)^{k-1} w(1)+\cdots-w(k-1)+w(k)\right) .
$$

Рассмотрим нечетные номера $k$, т.е. $k=2 m+1, m=0,1, \ldots$ Тогда

$$
y(2 m+2)=-y(1)-(w(1)-w(2)+\cdots-w(2 m)+w(2 m+1)) .
$$

Пусть $m^{0}$ - некоторьй номер, удовлетворяющий условию $|y(1)| \leqslant \bar{\rho}\left(2 m^{0}+1\right)$. Полагаем

$$
\begin{aligned}
w(2 i+1) & =-\frac{y(1)}{2 m^{0}+1}, \quad i=0,1, \ldots, m^{0}, \\
w(2 i) & =\frac{y(1)}{2 m^{0}+1}, \quad i=1, \ldots, m^{0}, \\
w(k) & =0, \quad k \geqslant 2 m^{0}+2 .
\end{aligned}
$$

Тогда

$$
y(k)=0, \quad k \geqslant 2 m^{0}+2 .
$$

Таким образом, существует номер $k^{0}$ такой, что $y(k)=0$ при $k \geqslant k^{0}+1$. Теперь последовательно рассмотрим уравнения системы (8). Согласно доказанному вьше существуют номера $k_{1}^{0}, k_{2}^{0}, \ldots, k_{l}^{0}$ и управления $w_{1}^{0}(\cdot), \ldots, w_{l}^{0}(\cdot)$ такие, что для решения $j$-го уравнения

$$
y_{j}^{0}(k+1)=\lambda_{j}^{0} y_{j}^{0}(k)-w_{j}^{0}(k), \quad j=1, \ldots, l,
$$

системы (8) имеем

$$
y_{j}^{0}(k)=0, \quad k \geqslant k_{1}^{0}+\cdots+k_{j}^{0}+1 \text {. }
$$

Пусть $m^{0}=k_{1}^{0}+\cdots+k_{l}^{0}$. Тогда $y_{j}^{0}(k)=0, j=1, \ldots, l$, при $k \geqslant m^{0}+1$. Следовательно, для решения $x_{1}^{0}(\cdot)$ имеем $x_{1}^{0}(k)=0$ при $k \geqslant m^{0}+1$.

2. Система, соответствующая клетке $J_{i}, 1 \leqslant i \leqslant l_{1}$. Каждая система

$$
x_{1}^{i}(k+1)=J_{i} x_{1}^{i}(k)-w_{1}^{i}(k), \quad k=m+1, m+2, \ldots,
$$


где $m$ - некоторое натуральное число, эквивалентна системе, имеющей в координатной форме следуюший вид:

$$
\left\{\begin{array}{rl}
y_{1}(k+1) & =\lambda y_{1}(k)+y_{2}(k)-w_{1}(k), \\
\ldots \ldots \ldots \ldots \ldots \ldots \ldots \ldots \ldots \ldots \ldots \ldots \ldots \ldots \ldots \ldots & \ldots \ldots \ldots \\
y_{r-1}(k+1) & =\lambda y_{r-1}(k)+y_{r}(k)-w_{r-1}(k), \\
y_{r}(k+1) & =\lambda y_{r}(k)-w_{r}(k),
\end{array} \quad k=m+1, m+2, \ldots,\right.
$$

где $m$ - некоторое натуральное число. Рассмотрим систему (11), начиная с последнего ее уравнения. Согласно доказанному в п. 1 можно выбрать номер $k_{r}$ и управление $w_{r}(\cdot)$ так, чтобы $y_{r}(k)=0, k \geqslant m+k_{r}+1$. Теперь рассмотрим предпоследнее уравнение системы (11)

$$
y_{r-1}(k+1)=\lambda y_{r-1}(k)+y_{r}(k)-w_{r-1}(k) \text {. }
$$

Полагаем $w_{r-1}(k)=0, k \leqslant m+k_{r}$. Рассмотрим уравнение (12) при $k \geqslant m+k_{r}+1$. Тогда с учетом того, что $y_{r}(k)=0, k \geqslant m+k_{r}+1$, имеем

$$
y_{r-1}(k+1)=\lambda y_{r-1}(k)-w_{r-1}(k), \quad k \geqslant m+k_{r}+1,
$$

где начальньм значением для переменного $y_{r-1}(k)$ будет число $y_{r-1}\left(m+k_{r}+1\right)$.

Аналогично рассмотренному выше случаю число $k_{r-1}$ и управление $w_{r-1}(\cdot)$ выберем так, чтобы $y_{r-1}(k)=0, k \geqslant m+k_{r}+k_{r-1}+1$. Дальше рассуждения проводятся аналогично случаям $r, r-1$.

Таким образом, существуют натуральные числа $k_{r}, k_{r-1}, \ldots, k_{1}$ и управления $w_{r}(\cdot)$, $w_{r-1}(\cdot), \ldots, w_{1}(\cdot)$ такие, что

$$
\begin{aligned}
& y_{r}(k)=0, \quad k \geqslant m+k_{r}+1, \\
& y_{r-1}(k)=0, \quad k \geqslant m+k_{r}+k_{r-1}+1, \\
& y_{1}(k)=0, \quad k \geqslant m+k_{r}+k_{r-1}+\cdots+k_{1}+1 .
\end{aligned}
$$

Теперь последовательно рассмотрим системы $(6)$ при $i=1,2, \ldots, l_{1}$. Согласно доказанному выше для $i$-й системы

$$
\left\{\begin{aligned}
& y_{1}^{i}(k+1)=\lambda y_{1}^{i}(k)+y_{2}^{i}(k)-w_{1}^{i}(k) \\
& \ldots \ldots \ldots \ldots \ldots \ldots \ldots \ldots \ldots \ldots \ldots \ldots \ldots \ldots \ldots \ldots \ldots \ldots \ldots \ldots \ldots \\
& y_{r_{i}-1}^{i}(k+1)=\lambda y_{r_{i}-1}^{i}(k)+y_{r_{i}}^{i}(k)-w_{r_{i}-1}^{i}(k) \\
& y_{r_{i}}^{i}(k+1)=\lambda y_{r_{i}}^{i}(k)-w_{r_{i}}^{i}(k)
\end{aligned}\right.
$$

существуют числа $k_{r_{i}}^{i}, k_{r_{i}-1}^{i}, \ldots, k_{1}^{i}$ и управления $w_{r_{i}}^{i}(\cdot), w_{r_{i}-1}^{i}(\cdot), \ldots, w_{1}^{i}(\cdot)$ такие, что вьполнены соотношения

$$
\begin{aligned}
& y_{r_{i}}^{i}(k)=0, \quad k \geqslant m^{i-1}+k_{r_{i}}^{i}+1, \\
& y_{r_{i}-1}^{i}(k)=0, \quad k \geqslant m^{i-1}+k_{r_{i}}^{i}+k_{r_{i}-1}^{i}+1, \\
& y_{1}^{i}(k)=0, \quad k \geqslant m^{i-1}+k_{r_{i}}^{i}+k_{r_{i}-1}^{i}+\cdots+k_{1}^{i}+1,
\end{aligned}
$$


где $m^{i}=m^{i-1}+k_{r_{i}}^{i}+k_{r_{i}-1}^{i}+\cdots+k_{1}^{i}, i=1,2, \ldots, l_{1}$. Тогда $x_{1}^{i}(k)=0$ при $k \geqslant m^{i}+1$, $i=1,2, \ldots, l_{1}$.

Теперь покажем возможность перевода фазовой точки $x_{2}^{i}$ в начало координат.

3. Система, соответствующая клетке $B_{0}$. Система

$$
x_{2}^{0}(k+1)=B_{0} x_{2}^{0}(k)-w_{2}^{0}(k), \quad k=m+1, m+2, \ldots,
$$

распадается на системы, имеющие в координатной форме следующий вид:

$$
\left\{\begin{array}{l}
y_{11}(k+1)=\alpha y_{11}(k)-\beta y_{21}(k)-w_{11}(k), \\
y_{21}(k+1)=\beta y_{11}(k)+\alpha y_{21}(k)-w_{21}(k),
\end{array} \quad k=m+1, m+2, \ldots\right.
$$

А. Случай, когда $\alpha^{2}+\beta^{2}<1$. Тогда номер $e^{0}$ выберем так, чтобы вьполнялось неравенство

$$
\left(\alpha^{2}+\beta^{2}\right)^{e^{0}} R^{2}(m+1) \leqslant \bar{\rho}^{2},
$$

где $R(k)=\sqrt{y_{11}^{2}(k)+y_{21}^{2}(k)}$. При $1 \leqslant k \leqslant m+e^{0}-1$ полагаем $w_{11}(k)=w_{21}(k)=0$. Тогда получаем

$$
\begin{gathered}
\left(\alpha y_{11}\left(m+e^{0}\right)-\beta y_{21}\left(m+e^{0}\right)\right)^{2}+\left(\beta y_{11}\left(m+e^{0}\right)+\alpha y_{21}\left(m+e^{0}\right)\right)^{2} \\
=\left(\alpha^{2}+\beta^{2}\right) R^{2}\left(m+e^{0}\right)=\cdots=\left(\alpha^{2}+\beta^{2}\right)^{e^{0}} R^{2}(m+1) \leqslant \bar{\rho}^{2}
\end{gathered}
$$

Тогда полагая

$$
\begin{aligned}
& w_{11}\left(m+e^{0}\right)=\alpha y_{11}\left(m+e^{0}\right)-\beta y_{21}\left(m+e^{0}\right), \\
& w_{21}\left(m+e^{0}\right)=\beta y_{11}\left(m+e^{0}\right)+\alpha y_{21}\left(m+e^{0}\right),
\end{aligned}
$$

получаем $y_{11}\left(m+e^{0}+1\right)=y_{21}\left(m+e^{0}+1\right)=0$.

Б. Случай, когда $\alpha^{2}+\beta^{2}=1$. Пусть номер $e^{0}$ удовлетворяет условию $\left(e^{0}-1\right) \bar{\rho}<$ $R(m+1) \leqslant e^{0} \bar{\rho}$ и пусть $\rho_{0}=R(m+1) / e^{0}$. Полагаем

$$
\begin{aligned}
& w_{11}(k)=\frac{\bar{\rho}_{0}}{R(k)}\left(\alpha y_{11}(k)-\beta y_{21}(k)\right), \\
& w_{21}(k)=\frac{\bar{\rho}_{0}}{R(k)}\left(\beta y_{11}(k)+\alpha y_{21}(k)\right),
\end{aligned}
$$

Тогда

$$
\begin{aligned}
& y_{11}(k+1)=\left(1-\frac{\bar{\rho}_{0}}{R(k)}\right)\left(\alpha y_{11}(k)-\beta y_{21}(k)\right), \\
& y_{21}(k+1)=\left(1-\frac{\bar{\rho}_{0}}{R(k)}\right)\left(\beta y_{11}(k)+\alpha y_{21}(k)\right) .
\end{aligned}
$$

Следовательно, с учетом $\alpha^{2}+\beta^{2}=1$ получаем

$$
R^{2}(k+1)=R^{2}(k)\left(1-\frac{\bar{\rho}_{0}}{R(k)}\right)^{2}\left(\alpha^{2}+\beta^{2}\right)=\left(R(k)-\bar{\rho}_{0}\right)^{2} .
$$


Следовательно,

$$
R\left(m+e^{0}+1\right)=R\left(m+e^{0}\right)-\bar{\rho}_{0}=\cdots=R(m+1)-e^{0} \bar{\rho}_{0}=0 .
$$

В каждом из случаев А, Б при $k \geqslant m+e^{0}+1$ полагаем $w_{11}(k)=w_{21}(k)=0$. Тогда $y_{11}(k)=y_{21}(k)=0, k \geqslant m+e^{0}+1$.

Теперь последовательно и попарно рассмотрим уравнения системы (13). Согласно доказанному вьше существуют номера $e_{1}^{0}, e_{2}^{0}, \ldots, e_{s_{0}}^{0}$ и управления $w_{11}(\cdot), w_{21}(\cdot), \ldots$, $w_{1, s_{0}}(\cdot), w_{2, s_{0}}(\cdot)$ такие, что для решения $j$-й пары уравнений

$$
n\left\{\begin{array}{l}
y_{1 j}(k+1)=\alpha_{j} y_{1 j}(k)-\beta_{j} y_{2 j}(k)-w_{1 j}(k), \\
y_{2 j}(k+1)=\beta_{j} y_{1 j}(k)+\alpha_{j} y_{2 j}(k)-w_{2 j}(k),
\end{array} \quad j=1,2, \ldots, s_{0},\right.
$$

системы (13) имеем

$$
y_{1 j}(k)=0, \quad y_{2 j}(k)=0, \quad k \geqslant m^{l_{1}}+e_{1}^{0}+\cdots+e_{j}^{0}+1, \quad j=1,2, \ldots, s_{0} .
$$

Пусть $n^{0}=m^{l_{1}}+e_{1}^{0}+\cdots+e_{s_{0}}^{0}$. Тогда $x_{2}^{0}(k)=0$ при $k \geqslant n^{0}+1$.

4. Система, соответствующая клетке $B_{j}, 1 \leqslant j \leqslant l_{2}$. При каждом $j, 1 \leqslant j \leqslant l_{2}$, система (7) эквивалентна некоторой системе, имеющей вид

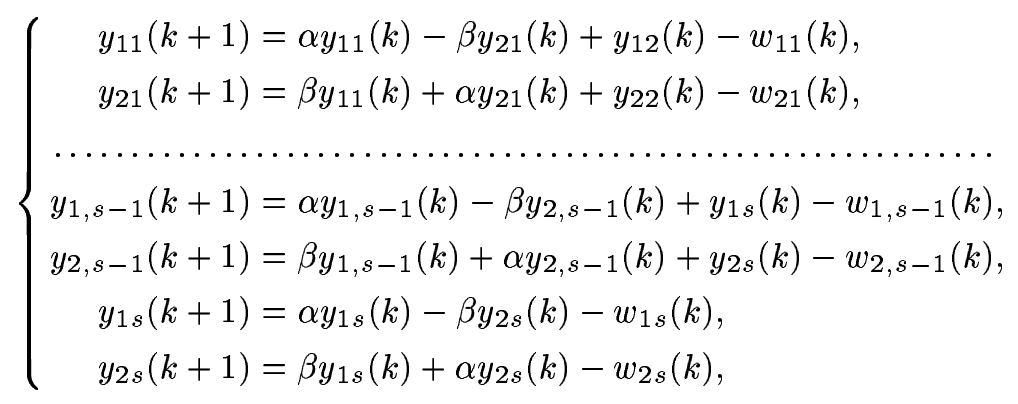

где $k=m+1, m+2, \ldots, m$ - некоторое натуральное число. Рассмотрим систему (14), начиная с последней пары ее уравнений. Полагаем $w_{1 j}(k)=w_{2 j}(k)=0, j=1, \ldots, s$, при $k=1,2, \ldots, m$.

Согласно доказанному в п. 3 можно выбрать номер $e_{s}$ и управления $w_{1 s}(\cdot), w_{2 s}(\cdot)$ так, чтобы $y_{1 s}(k)=y_{2 s}(k)=0, k \geqslant m+e_{s}+1$.

Теперь рассмотрим предпоследнюю пару уравнений системы (14)

$$
\left\{\begin{array}{l}
y_{1, s-1}(k+1)=\alpha y_{1, s-1}(k)-\beta y_{2, s-1}(k)+y_{1 s}(k)-w_{1, s-1}(k) \\
y_{2, s-1}(k+1)=\beta y_{1, s-1}(k)+\alpha y_{2, s-1}(k)+y_{2 s}(k)-w_{2, s-1}(k) .
\end{array}\right.
$$

Полагаем $w_{1, s-1}(k)=0, w_{2, s-1}(k)=0, k \leqslant m+e_{s}$. Рассмотрим систему (15) при $k \geqslant m+e_{s}+1$. Тогда с учетом того, что $y_{1 s}(k)=0, y_{2 s}(k)=0, k \geqslant m+e_{s}+1$, имеем

$$
\left\{\begin{array}{l}
y_{1, s-1}(k+1)=\alpha y_{1, s-1}(k)-\beta y_{2, s-1}(k)-w_{1, s-1}(k), \\
y_{2, s-1}(k+1)=\beta y_{1, s-1}(k)+\alpha y_{2, s-1}(k)-w_{2, s-1}(k),
\end{array}\right.
$$


где начальным значением для переменных $y_{1, s-1}(k)$ и $y_{2, s-1}(k)$ будут числа $y_{1, s-1}(m+$ $\left.e_{s}+1\right)$ и $y_{2, s-1}\left(m+e_{s}+1\right)$.

Аналогично рассмотренному вьше случаю число $e_{r-1}$ и управления $w_{1, s-1}(\cdot)$ и $w_{2, s-1}(\cdot)$ выберем так, чтобы $y_{1, s-1}(k)=y_{2, s-1}(k)=0$ при $k=m+e_{s}+e_{s-1}+1$. При $k \geqslant m+e_{s}+e_{s-1}+1$ полагаем $w_{1, s-1}(k)=0, w_{2, s-1}(k)=0$. Тогда при таких $k$ имеем $y_{1, s-1}(k)=y_{2, s-1}(k)=0$. Дальше рассуждения проводятся аналогично случаям $s$, $s-1$.

Таким образом, сушествуют числа $e_{s}, e_{s-1}, \ldots, e_{1}$ и управления $w_{1 s}(\cdot), w_{2 s}(\cdot), \ldots$, $w_{11}(\cdot), w_{21}(\cdot)$ такие, что

$$
y_{1 j}(k)=y_{2 j}(k)=0, \quad k \geqslant m+e_{s}+\cdots+e_{j}+1, \quad j=s, s-1, \ldots, 1 .
$$

Теперь последовательно рассмотрим системы (7) при $j=1,2, \ldots, l_{2}$. Согласно доказанному вьше для $j$-й системы существуют числа $e_{s_{j}}^{j}, e_{s_{j}-1}^{j}, \ldots, e_{1}^{j}$ и управления $w_{1, s_{j}}^{j}(\cdot), w_{2, s_{j}}^{j}(\cdot), \ldots, w_{11}^{j}(\cdot), w_{21}^{j}(\cdot)$ такие, что выполнены соотношения $x_{2}^{j}(k)=0$, $k \geqslant n^{j}+1$, где $n^{j}=n^{j-1}+e_{s_{j}}^{j}+e_{s_{j}-1}^{j}+\cdots+e_{1}^{j}+1, j=1,2, \ldots, l_{2}$. Таким образом, $x(k)=0, k \geqslant n^{l_{2}}+1$.

5. Завершение доказательства леммы 1. Пусть

$$
w(\cdot)=(w(1), w(2), \ldots, w(k), \ldots), \quad|w(k)| \leqslant \bar{\rho}
$$

- последовательность, переводящая в системе (5) фазовую точку $x$ из начального положения $x_{0}=S^{-1} z_{0}$ в начало координат за $N\left(x_{0}\right)$ шагов, т.е. $x\left(N\left(x_{0}\right)\right)=0$. Тогда $z\left(N\left(x_{0}\right)\right)=S x\left(N\left(x_{0}\right)\right)=0$, т.е. управление $\bar{w}(k)=S w(k), k=1,2, \ldots$, переводит фазовую точку $z$ из начального положения $z_{0}$ в начало координат за $N\left(x_{0}\right)$ шагов.

Покажем допустимость управления $\bar{w}(k)$.

Поскольку $S$ - неособенная действительная матрица, то $S^{\prime} S$ - симметрическая положительно определенная матрица, где $S^{\prime}$ - транспонирование матрищы $S$. Согласно утверждению 1 (см. приложение) для наибольшего собственного значения $\lambda$ матрицы $S^{\prime} S$ верно неравенство

$$
x^{\prime} S^{\prime} S x \leqslant \lambda|x|^{2}, \quad x \in \mathbb{R}^{n}
$$

Taк как $\bar{w}(k)=S w(k)$, то

$$
|\bar{w}(k)|^{2}=w^{\prime}(k) S^{\prime} S w(k) \leqslant \lambda|w(k)|^{2} .
$$

Если тогда полагаем $\bar{\rho}=(\rho-\sigma) / \sqrt{\lambda}$, то

$$
|\bar{w}(k)| \leqslant \sqrt{\lambda}|w(k)| \leqslant \sqrt{\lambda} \bar{\rho}=\rho-\sigma .
$$

Лемма 1 доказана. 
ДоКАЗАТЕЛЬСТВО ТЕОРЕМЫ 1. Стратегию преследующего построим так:

$$
u(k)=v(k)+\bar{w}(k), \quad k=1,2, \ldots,
$$

где $\bar{w}(\cdot)=(\bar{w}(1), \bar{w}(2), \ldots, \bar{w}(k), \ldots),|\bar{w}(k)| \leqslant \rho-\sigma,-$ управление, переводящее в системе (4) фазовую точку $z$ из начального положения $z_{0}$ в начало координат. Допустимость этой стратегии очевидна. Тогда получаем систему (4). Согласно лемме 1 в системе (4) из начального положения $z_{0}$ возможен перевод фазовой точки $z$ в начало координат. Теорема 1 доказана.

\section{3. Решение задачи 2.}

ТЕОрема 2. Если все собственные числа матрицы $С$ по модулю не превосходят единиць, то в игре (1), (3) из любого начального положсния $z_{0}$ возможно завершение преследования за некоторое конечное число шагов $N\left(z_{0}\right)$.

ДокАЗАтЕЛьСтво. Рассмотрим систему (1). Пусть $r, 0<r<\rho,-$ произвольное фиксированное число. Полагаем

$$
u(k)= \begin{cases}v(k)+w(k), & |v(k)| \leqslant \rho-r \\ 0, & |v(k)|>\rho-r\end{cases}
$$

где $w(k) \leqslant r$.

Если все время $|v(k)| \leqslant \rho-r, k \geqslant 1$, то $u(k)=v(k)+w(k), k \geqslant 1$, и из уравнения (1) получаем уравнение

$$
z(k+1)=C z(k)-w(k) .
$$

Тогда согласно решению задачи 1 существуют число $N_{1}=N_{1}(z(1))$ и управление $w(\cdot)$ такие, что $z\left(N_{1}+1\right)=0$.

Если на некотором шаге $k_{1}$ выполнено неравенство $\left|v\left(k_{1}\right)\right|>\rho-r$, то рассмотрим систему

$$
z(k+1)=C z(k)-u(k)+v(k)
$$

при $k \geqslant k_{1}+2$ с начальньм условием $z\left(k_{1}+1\right)=C z\left(k_{1}\right)+v\left(k_{1}\right)$.

Если теперь все время $|v(k)| \leqslant \rho-r, k \geqslant k_{1}+1$, то согласно решению задачи 1 существуют число $N_{2}=N_{2}\left(z\left(k_{1}\right)\right)$ и управление $w(\cdot)$ такие, что $z\left(N_{2}+1\right)=0$ и т.д.

Таким образом, для предотвращения завершения игры должны на некоторых шагах $k_{1}, k_{2}, \ldots$ выполняться неравенства

$$
\left|v\left(k_{i}\right)\right|>\rho-r, \quad i=1,2, \ldots
$$

Так как последовательность $v(1), v(2), \ldots$ удовлетворяет ограничению (3), неравенства (16) могут вьполняться лишь конечное число раз. Затем все время вьполняется неравенство $|v(k)| \leqslant \rho-r$. Тогда опять согласно решению задачи 1 существуют число $N_{0}$ и управление $w(\cdot)$ такие, что $z\left(N_{0}+1\right)=0$, т.е. в игре $(1),(3)$ возможно завершение преследования за конечное число шагов. Теорема 2 доказана.

4. Невозможность завершения преследования из некоторых точек пространства. Теперь рассмотрим систему (4). 
Лемма 2. Если матрииа $C$ имеет собственное число $\mu$ по модулю больие единицы, то в системе (4) $c|\bar{w}(k)| \leqslant \hat{\rho}$ из некоторых начальных положсений $z(1)$ невозможен перевод фазовой точки $z$ в начало координат.

ДокАЗАТЕЛЬСТво. С помощью действительного неособенного преобразования $z=S x$ систему (4) приведем к виду

$$
x(k+1)=J x(k)-\omega(k), \quad \omega(k)=S^{-1} \bar{w}(k),
$$

где $J=S^{-1} C S$ - жорданова форма матрицы $C$.

Достаточно показать невозможность перевода в системе (17) фазовой точки $z$ в начало координат из некоторых начальных положений $x(1)$ при $|\omega(k)| \leqslant \rho_{1}$, где $\rho_{1}$ - произвольное положительное число. Рассмотрим два случая.

А. Пусть $\mu, \mu>1,-$ вешественное собственное число матрицы $C$. Тогда система (17) содержит скалярное уравнение вида

$$
y(k+1)=\mu y(k)-\omega(k),
$$

где $y$ и $\omega-$ координаты векторов $x$ и $w$ соответственно. Для доказательства теоремы достаточно показать, что можно выбрать начальное положение $y(1)$ так, чтобы $y(k) \neq 0$, $k=1,2, \ldots$. Действительно,

$$
\begin{aligned}
|y(k+1)| & \geqslant\left|\mu^{k} y(1)\right|-\left(\left|\mu^{k-1}\right||\omega(1)|+\cdots+|\mu||\omega(k-1)|+|\omega(k)|\right) \\
& \geqslant\left|\mu^{k} y(1)\right|-\rho_{1}\left(\left|\mu^{k-1}\right|+\cdots+|\mu|+1\right)=|\mu|^{k}\left(|y(1)|-\frac{\rho_{1}\left(1-|\mu|^{-k}\right)}{|\mu|-1}\right) .
\end{aligned}
$$

Если

$$
|y(1)|>\frac{\rho_{1}\left(1-|\mu|^{-k}\right)}{|\mu|-1},
$$

то ясно, что выполняется неравенство $|y(k+1)|>0, k=1,2, \ldots$

Б. Пусть $\mu=\alpha+\beta i, \beta \neq 0,|\mu|>1$. Тогда система (17) содержит систему вида

$$
\left\{\begin{array}{l}
y_{1}(k+1)=\alpha y_{1}(k)-\beta y_{2}(k)-\omega_{1}(k), \\
y_{2}(k+1)=\beta y_{1}(k)+\alpha y_{2}(k)-\omega_{2}(k) .
\end{array}\right.
$$

Обозначив

$$
y(k)=\sqrt{y_{1}^{2}(k)+y_{2}^{2}(k)}, \quad \omega(k)=\sqrt{\omega_{1}^{2}(k)+\omega_{2}^{2}(k)},
$$

получаем

$$
\begin{aligned}
y(k+1) & =\left[\left(\alpha y_{1}(k)-\beta y_{2}(k)-\omega_{1}(k)\right)^{2}+\left(\beta y_{1}(k)+\alpha y_{2}(k)-\omega_{2}(k)\right)^{2}\right]^{1 / 2} \\
& \geqslant\left[\left(\alpha y_{1}(k)-\beta y_{2}(k)\right)^{2}+\left(\beta y_{1}(k)+\alpha y_{2}(k)\right)^{2}\right]^{1 / 2}-\left(\omega_{1}^{2}(k)+\omega_{2}^{2}(k)\right)^{1 / 2} \\
& \geqslant|\mu| y(k)-\omega(k) .
\end{aligned}
$$

Итак $y(k+1) \geqslant|\mu| y(k)-\omega(k)$. Дальше рассуждения проводятся аналогично случаю А. Лемма 2 доказана.

Следуюшая теорема показывает, что условие: все собственные числа матрицы $C$ не превосходят единицы, является и необходимым условием для завершения преследования из всех точек пространства. 
Теорема 3. Если матрица $C$ имеет собственное число $\mu$ по модулю больше единицы, то в игре (1), (2) (соответственно (1), (3)) из некоторых начальных положений $z_{0}$ невозможсно завершение преследования.

Для доказательства теоремы 3 достаточно полагать в системе (1) $v(k)=0, k=1$, $2, \ldots$ и для полученной системы

$$
z(k+1)=C z(k)-u(k), \quad|u(k)| \leqslant \rho,
$$

применить лемму 2.

Приложение. Здесь мы приведем некоторые известные факты и утверждения [13], [14] в нужной для нас форме.

Жорданова форма матрицы. Известно, что матрица $C$ подобна некоторой матрице $J$, если существует неособенная действительная матрица $S$ такая, что $C=S J S^{-1}$, где

$$
J=\left(\begin{array}{ccccccc}
J_{0} & & & & & & \\
& J_{1} & & & & & \\
\cdot & \cdot & \cdot & \cdot & \cdot & \cdot & \cdot \\
& & & J_{l_{1}} & & & \\
& & & & B_{0} & & \\
& & & & & B_{1} & \\
\cdot & \cdot & \cdot & \cdot & \cdot & \cdot & \cdot \\
0 & & & & & & B_{l_{2}}
\end{array}\right),
$$

т.е. $J=\operatorname{diag}\left\{J_{0}, J_{1}, \ldots, J_{l_{1}}, B_{0}, B_{1}, \ldots, B_{l_{2}}\right\}, J_{0}, J_{1}, \ldots, J_{l_{1}}$ соответствуют действительным собственньм числам матрицы $C$, а $B_{0}, B_{1}, \ldots, B_{l_{2}}$ - собственным числам, имеюшим ненулевую мнимую часть:

$$
J_{0}=\left(\begin{array}{cccc}
\lambda_{1} & 0 & \ldots & 0 \\
0 & \lambda_{2} & \ldots & 0 \\
. & . & . & . \\
0 & 0 & \ldots & \lambda_{l}
\end{array}\right), \quad J_{i}=\left(\begin{array}{ccccc}
\lambda_{i} & 1 & 0 & \ldots & 0 \\
0 & \lambda_{i} & 1 & \ldots & 0 \\
. & . & . & . & . \\
0 & 0 & 0 & \ldots & \lambda_{i}
\end{array}\right),
$$

$i=1, \ldots, l_{1}, B_{0}=\operatorname{diag}\left\{T_{1}, T_{2}, \ldots, T_{s}\right\}$,

$$
B_{j}=\left(\begin{array}{ccccc}
T_{j} & E_{2} & O_{2} & \ldots & O_{2} \\
O_{2} & T_{j} & E_{2} & \ldots & O_{2} \\
\cdot & \cdot & \cdot & \cdot & \cdot \\
O_{2} & O_{2} & O_{2} & \ldots & T_{j}
\end{array}\right), \quad T_{j}=\left(\begin{array}{cc}
\alpha_{j} & -\beta_{j} \\
\beta_{j} & \alpha_{j}
\end{array}\right),
$$

$j=1, \ldots, l_{2}, E_{2}$ - единичная $(2 \times 2)$-матрища, $O_{2}$ - нулевая $(2 \times 2)$-матрица, $\alpha_{j}$ и $\beta_{j}-$ вещественные и мнимые части собственных чисел матрищы $C$.

УТВеРЖДЕНИЕ (см. [13, с. 252]). Если А - вещественная симметрическая матрица, а $\lambda^{-}(A)$ и $\lambda^{+}(A)$ - ее минимальное и максимальное собственные числа соответственно, то для всех $x \in \mathbb{R}^{n}$ имеют место неравенства

$$
\lambda^{-}(A)|x|^{2} \leqslant x^{\prime} A x \leqslant \lambda^{+}(A)|x|^{2} .
$$




\section{СПИСОК ЦИТИРОВАННОЙ ЛИТЕРАТУРЫ}

[1] Айзекс Р. Дифференциальные игры. М.: Мир, 1967.

[2] Понтрягин Л.С. Избранные труды. М.: Наука, 1988.

[3] Красовский Н. Н. Управление динамической системой. М.: Наука, 1985.

[4] Субботин А. И., Ченцов А. Г. Оптимизация гарантии в задачах управления. М.: Наука, 1981.

[5] Петросян Л. А. Дифференциальные игры преследования. Л.: Изд-во ЛГУ, 1977.

[6] Никольский М. С. О задаче преследования при различных ограничениях на управления догоняющего и убегающего // Теория оптимальных решений. Киев: Изд-е Ин-та кибернетики, 1975. C. $59-66$.

[7] Сатимов Н. Ю., Рихсиев Б. Б. Методы решения задачи уклонения от встречи в математической теории управления. Ташкент: Фан, 2000.

[8] Сатимов Н.Ю., Рихсиев Б. Б., Хамдамов А. А. О задаче преследования для линейных дифференциальных и дискретных игр многих лиц // Матем. сб. 1982. Т. 118. № 4. C. $456-469$.

[9] Сатимов Н. Ю., Фазылов А. З., Хамдамов А. А. О задачах преследования и уклонения в дифференциальных и дискретных играх многих лиц с интегральными ограничениями // Дифференц. уравнения. 1984. Т. 20. № 8. С. 1388-1396.

[10] Рихсиев Б. Б. Дифференциальные игры с простыми движениями. Ташкент: Фан, 1989.

[11] Ибрагимов Г. И. К задаче группового преследования с интегральными ограничениями на управления игроков // Матем. заметки. 2001. Т. 70. № 2. С. 201-212.

[12] Ибрагимов Г. И. Игровая задача на выпуклом замкнутом множестве // Матем. труды. 2001. Т. 4. № 2. С. 96-112.

[13] Треногин В. А. Функциональный анализ. М.: Наука, 1980.

[14] Гантмахер Ф.Р. Теория матриц. М.: Наука, 1988.

Университет мировой экономики и дипломатии, г. Ташкент

Поступило 30.01 .2004 